\title{
Forecasting Models Evaluation Using A Slacks-Based Context-Dependent DEA Framework
}

\author{
Jamal Ouenniche, University of Edinburgh, Edinburgh, United Kingdom \& Business School, ESC Rennes, France \\ Bing Xu, Heriot-Watt University, UK \\ Kaoru Tone, National Graduate Institute for Policy Studies, Japan
}

\begin{abstract}
$X u$ and Ouenniche (2012a) proposed an input-oriented radial super-efficiency Data Envelopment Analysis (DEA) based model to address a common methodological issue in the evaluation of competing forecasting models; namely, ranking models based on a single performance measure at a time, which typically leads to conflicting ranks. However, their approach suffers from a number of issues. In this paper, we overcome these issues by proposing a slacks-based context-dependent DEA framework and use it to rank forecasting models of oil prices' volatility.
\end{abstract}

Keywords: Forecasting Crude Oil Prices' Volatility; Performance Evaluation; Orientation-Free DEA

\section{INTRODUCTION}

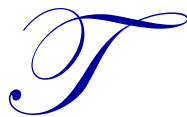

he design of quantitative models for forecasting continuous variables in a wide range of application areas has attracted the attention of a large number of academics and professionals for some time; however, the performance evaluation of competing forecasting models has not received as much attention. Nowadays, although most published research involve using several performance criteria and measures to compare models, the performance evaluation exercise remains of a unidimensional nature; that is, models are ranked by a single measure and typically the obtained rankings are conflicting. Therefore, one cannot make an informed decision as to which model performs better under several criteria and their measures. To the best of our knowledge, the only papers that both raised concerns about this methodological issue and addressed it are the ones by Xu and Ouenniche $(2011,2012 \mathrm{a}, 2012 \mathrm{~b})$. A super-efficiency data envelopment analysis model has been proposed by Xu and Ouenniche (2011) to devise a multi-criteria ranking of competing forecasting models of oil prices' volatility; however, their approach suffers from the following issues. First, in many applications such as the ranking of forecasting models, the choice of an orientation is irrelevant. Second, under the variable returns-to-scale assumption, input-oriented scores can be different from output-oriented ones, which may lead to different rankings. Third, radial DEA models could only take account of technical efficiency and ignore potential slacks in inputs and outputs and thus may over-estimate efficiency scores. Fourth, radial super-efficiency DEA models may be infeasible for some efficient decision making units (DMUs) and would lead to unresolved ties. Finally, within a super-efficiency DEA framework, super-efficiency scores are used to rank order the efficient DMUs; however, the reference set changes from one efficient DMU evaluation to another, which in some contexts might be viewed as "unfair" benchmarking. In this paper, we overcome these issues by proposing a slacks-based context-dependent DEA (CDEA) framework (Morita, Hirokawa, \& Zhu, 2005; Seiford \& Zhu, 2003) for assessing the relative performance of competing volatility forecasting models.

The remainder of this paper is organized as follows. In Section 2, we describe the proposed slacks-based CDEA framework to evaluate the relative performance of competing volatility forecasting models. In Section 3, we report on our empirical findings. Section 4 concludes the paper. 


\section{A SLACKS-BASED CDEA MODEL FOR ASSESSING FORECASTING MODELS}

In this paper, we propose a slacks-based CDEA framework to assess the relative performance of competing forecasting models for crude oil prices' volatility. The proposed framework is a three-stage process which could be summarized as follows:

Stage 1 - Returns-to-Scale (RTS) Analysis: Perform RTS analysis to find out whether to solve a DEA model under constant returns-to-scale (CRS) conditions, variable returns-to-scale (VRS) conditions, increased returns-to-scale (IRS) conditions, or decreased returns-to-scale (DRS) conditions - see Banker, Cooper, Seiford, Thrall, and Zhu (2004) and Majid (2012) for details.

Stage 2 - Classification of DMUs: Use the following algorithm to partition the set of DMUs into several levels of best-practice frontiers or evaluation contexts, say $L$ :

\section{Initialization Step}

Initialize the performance level counter $\ell$ to 1 and the set of DMUs to evaluate at level $\ell$, say $J^{\ell}$, to $\left\{D M U_{k}, k=1, \ldots, n\right\}$. Use the relevant DEA model to evaluate $J^{\ell}$ and set the $\ell^{\text {th }}$-level best-practice frontier $E^{\ell}$ accordingly; that is, $E^{\ell}=\left\{k \in J^{\ell} \mid\right.$ Efficiency Score $\left.\rho_{k}^{\ell}=1\right\}$. Exclude the current performance level best-practice frontier $E^{\ell}$ from the set of DMUs to evaluate next; that is, set $J^{\ell+1}=J^{\ell}-E^{\ell}$, increment $\ell$ by 1 and proceed to the iterative step.

\section{Iterative Step}

While $J^{\ell} \neq \varnothing$ Do

\{

Use the relevant DEA model to evaluate $J^{\ell}$, set the $\ell^{\text {th }}$-level best-practice frontier $E^{\ell}$ accordingly, set $J^{\ell+1}=J^{\ell}-E^{\ell}$, and increment $\ell$ by 1 ;

$\underline{\underline{3}}$

where the relevant DEA model to use is the slacks-based measure (SBM) model of Tone (2001):

$$
\begin{array}{ll}
\text { Min } & \rho_{k}^{\ell}=\left(1-\frac{1}{m} \sum_{i=1}^{m} \frac{s_{i, k}^{-}}{x_{i, k}}\right) /\left(1+\frac{1}{s} \sum_{r=1}^{s} \frac{s_{r, k}^{+}}{y_{r, k}}\right) \\
\text { s.t.: } & \sum_{j \in J^{\ell}} \lambda_{j} x_{i, j}+s_{i, k}^{-}=x_{i, k} ; \forall i \\
& \sum_{j \in J^{\ell}} \lambda_{j} y_{r, j}-s_{r, k}^{+}=y_{r, k} ; \forall r \\
& \lambda_{j} \geq 0, \forall j \in J^{\ell} ; s_{i, k}^{-} \geq 0, \forall i ; s_{r, k}^{+} \geq 0, \forall r
\end{array}
$$

where the $i^{\text {th }}$ input and $r^{\text {th }}$ output of $D M U_{j}(j=1, \ldots, n)$ are denoted by $x_{i, j}(i=1, \ldots, m)$ and $y_{r, j}(r=1, \ldots, s)$, respectively, $\lambda_{j}$ is the weight assigned to $D M U_{j}$ in constructing its ideal benchmark, $s_{i, k}^{-}$and $s_{r, k}^{+}$are slack variables associated with the first and the second sets of constraints, respectively, and $\rho_{k}^{\ell}$ denotes the SBM efficiency score of $D M U_{k}$ achieved at performance level $\ell$. If the optimal value of $\rho_{k}^{\ell}=1$, then $D M U_{k}$ is classified as efficient; otherwise $D M U_{k}$ is classified as inefficient. Note that model 1 above is solved as it is if stage 1 reveals that the CRS conditions hold; otherwise, one would have to impose one of the following additional constraints depending on whether VRS, IRS, or DRS conditions prevail, respectively: 


$$
\sum_{j \in J^{\ell}} \lambda_{j}=1 ; \sum_{j \in J^{\ell}} \lambda_{j} \geq 1 ; \sum_{j \in J^{\ell}} \lambda_{j} \leq 1
$$

Obviously, once DMUs have been partitioned into $L$ efficient frontiers with different levels of performance, one could rank order them from best to worst starting with $1^{\text {st }}$-level efficient frontier DMUs as best and ending with the $L^{\text {th }}$-level efficient frontier DMUs as worst. Note that ties might exist between DMUs on the same efficient frontier and the next stage is designed to break those ties.

Stage 3 - Break Efficiency Ties: First, for each efficient frontier $E^{\ell}(\ell=2, \ldots, \mathrm{L})$, compute relative progress scores $\delta_{k}^{1} \mathrm{~s}$ with respect to the best evaluation context, ${ }^{1} E^{1}$, by solving the following model for each $D M U_{k} \in E^{\ell}$ and rank order DMUs on efficient frontier $E^{\ell}$ according to the values of these scores:

$$
\begin{array}{ll}
\text { Min } & \delta_{k}^{1}=\left(1-\frac{1}{m} \sum_{i=1}^{m} \frac{t_{i, k}^{-}}{x_{i, k}}\right) /\left(1+\frac{1}{s} \sum_{r=1}^{s} \frac{t_{r, k}^{+}}{y_{r, k}}\right) \\
\text { s.t.: } & \sum_{j \in E^{1}} \lambda_{j} x_{i, j} \geq x_{i, k}-t_{i, k}^{-} ; \forall i \\
& \sum_{j \in E^{1}} \lambda_{j} y_{r, j} \leq y_{r, k}+t_{r, k}^{+} ; \forall r \\
& \lambda_{j} \geq 0, \forall j \in E^{1} ; t_{i, k}^{-} \geq 0, \forall i ; t_{r, k}^{+} \geq 0, \forall r \geq 0
\end{array}
$$

where $t_{i, k}^{-}$(respectively, $t_{r, k}^{+}$) denotes the amount by which input $i$ (respectively, output $r$ ) of $D M U_{k}$ should be decreased (respectively, increased) to reach the efficient frontier corresponding to evaluation context $E^{1}$. Second, for DMUs belonging to the best efficient frontier $E^{1}$, compute relative attractiveness scores $\gamma_{k}^{2} \mathrm{~s}$ with respect to the second best evaluation context, ${ }^{2} E^{2}$, by solving the following model for each $D M U_{k} \in E^{1}$ and rank order DMUs on the best efficient frontier according to the values of these scores:

$$
\begin{array}{ll}
\operatorname{Max} & \gamma_{k}^{2}=\left(1-\frac{1}{m} \sum_{i=1}^{m} \frac{t_{i, k}^{+}}{x_{i, k}}\right) /\left(1+\frac{1}{s} \sum_{r=1}^{s} \frac{t_{r, k}^{-}}{y_{r, k}}\right) \\
\text { s.t.: } & \sum_{j \in E^{2}} \lambda_{j} x_{i, j} \leq x_{i, k}+t_{i, k}^{+} ; \forall i \\
& \sum_{j \in E^{2}} \lambda_{j} y_{r, j} \geq y_{r, k}-t_{r, k}^{-} ; \forall r \\
& \lambda_{j} \geq 0, \forall j \in E^{2} ; t_{i, k}^{+} \geq 0, \forall i ; t_{r, k}^{-} \geq 0, \forall r
\end{array}
$$

where $t_{i, k}^{+}$(respectively, $t_{r, k}^{-}$) denotes the amount by which input $i$ (respectively, output $r$ ) of $D M U_{k} \in E^{1}$ should be increased (respectively, decreased) to reach the frontier corresponding to evaluation context $E^{2}$.

In the next section, we use the proposed procedure to rank order competing forecasting models of crude oil prices' volatility and report on our empirical findings.

\footnotetext{
${ }^{1}$ The rationale behind this choice is to set a common global target for all lower level efficient frontiers for the sake of fairness in benchmarking.

${ }^{2}$ The rationale behind this choice is to compare the most efficient DMUs with those that have the closest performance.
} 


\section{EMPIRICAL INVESTIGATION AND RESULTS}

For comparison purposes with the results obtained by Xu and Ouenniche (2012a), we use the same data, inputs, outputs, and forecasting models. ${ }^{3}$ Note that RTS analysis revealed that VRS conditions hold and therefore models 1, 3, and 4 are augmented with the following constraint: $\sum_{j \in J^{\ell}} \lambda_{j}=1$. Table 1 (respectively, Tables 2 and 4) provide the unidimensional (respectively, multidimensional) rankings of 14 forecasting models of crude oil prices' volatility based on 9 measures of 3 criteria: biasedness, goodness-of-fit, and correct sign. Table 1 is a typical output presented by most existing forecasting studies - these unidimensional rankings are devised as follows: models are ranked from best to worst using the relevant measure of each of the criteria under consideration. Notice that different criteria led to different unidimensional rankings, which provides evidence of the problem resulting from the use of a unidimensional approach in a multicriteria setting. Table 2 summarizes multidimensional rankings, where the models are ranked from best to worst based on the corresponding super-efficiency scores obtained using both input-oriented and output-oriented radial super-efficiency DEA models. Notice that, under VRS conditions, the rankings of inputand output-oriented analyses are different and the rankings of output-oriented analysis show more ties. Table 3 provides the efficient frontiers obtained with SBM-CDEA. These results suggest that the best and the worst efficient frontiers are insensitive to adjusting biasedness measures for volatility. Note that any rankings based on these efficient frontiers would lead to a large number of ties. In order to break these ties, we use relative progress and attractiveness scores obtained by solving models 3 and 4 , respectively, which results in the multidimensional rankings provided in Table 4 where models are ranked from best to worst based on these relative scores. Notice that Tables 2 and 4 reveal that the multicriteria rankings of models obtained by input- and output-oriented super-efficiency DEA analyses and SBM-CDEA analysis are different. These differences are due to the fact that input-oriented analysis minimizes inputs for fixed amounts of output and output-oriented analysis maximizes outputs for fixed amounts of input, whereas orientation-free analysis optimizes both inputs and outputs simultaneously. In addition, oriented super-efficiency analyses only take account of technical efficiency, whereas orientation-free CDEA analysis takes account of an additional performance component; namely, slacks. In fact, for our data set - see Table 4, the efficient model SMA20 maintained its best position in the rankings regardless of the type of DEA analysis, because it is always on the best efficient frontier and has zero slacks regardless of the performance measures used. As to the rankings of the remaining models, there are differences that are mainly due to the presence of slacks and the nature of benchmarks.

\footnotetext{
${ }^{3}$ Inputs (respectively, outputs) consist of performance metrics to be minimized (respectively, maximized) according to the principle of the less (respectively, the more) the better - for space limitations, description of performance metrics and forecasting models are provided in Xu and Ouenniche (2012).
} 


\begin{tabular}{|c|c|c|c|}
\hline \multicolumn{4}{|r|}{ Ranked From Best to Worst } \\
\hline \multirow{2}{*}{ Biasedness } & Mean Error (ME) & \multicolumn{2}{|c|}{$3 \rightarrow 5 \rightarrow 11 \rightarrow 9 \rightarrow 14 \rightarrow 10 \rightarrow 12 \rightarrow 4 \rightarrow 13 \rightarrow 6 \rightarrow 2 \rightarrow 8 \rightarrow 7 \rightarrow 1$} \\
\hline & Mean Volatility-Scaled Errors (MVolScE) & \multicolumn{2}{|c|}{$3 \rightarrow 5 \rightarrow 9 \& 10 \& 11 \& 12 \& 14 \rightarrow 4 \rightarrow 6 \& 13 \rightarrow 2 \rightarrow 8 \rightarrow 7 \rightarrow 1$} \\
\hline \multirow{6}{*}{ Goodness-of-fit } & Mean Absolute Error (MAE) & \multicolumn{2}{|c|}{$8 \rightarrow 5 \rightarrow 6 \rightarrow 3 \rightarrow 9 \& 10 \rightarrow 11 \rightarrow 12 \rightarrow 7 \rightarrow 14 \rightarrow 13 \rightarrow 4 \rightarrow 1 \rightarrow 2$} \\
\hline & Mean Absolute Volatility-Scaled Errors (MAVolScE) & \multicolumn{2}{|c|}{$5 \& 8 \rightarrow 3 \& 6 \rightarrow 9 \& 10 \& 11 \& 12 \rightarrow 7 \& 14 \rightarrow 13 \rightarrow 4 \rightarrow 1 \rightarrow 2$} \\
\hline & Mean Squared Error (MSE) & \multicolumn{2}{|c|}{$14 \rightarrow 13 \rightarrow 10 \rightarrow 12 \rightarrow 11 \rightarrow 9 \rightarrow 5 \rightarrow 3 \rightarrow 6 \rightarrow 4 \rightarrow 8 \rightarrow 7 \rightarrow 2 \rightarrow 1$} \\
\hline & Mean Squared Volatility-Scaled Errors (MSVolScE) & \multicolumn{2}{|c|}{$14 \rightarrow 13 \rightarrow 10 \rightarrow 11 \& 12 \rightarrow 9 \rightarrow 5 \rightarrow 3 \rightarrow 6 \rightarrow 4 \rightarrow 8 \rightarrow 7 \rightarrow 2 \rightarrow 1$} \\
\hline & Mean Mixed Error Under-estimation penalized (MMEU) & \multicolumn{2}{|c|}{$14 \rightarrow 3 \rightarrow 5 \rightarrow 10 \rightarrow 13 \rightarrow 12 \rightarrow 9 \rightarrow 11 \rightarrow 4 \rightarrow 6 \rightarrow 8 \rightarrow 7 \rightarrow 2 \rightarrow 1$} \\
\hline & Mean Mixed Error Over-estimation penalized (MMEO) & \multicolumn{2}{|c|}{$1 \rightarrow 2 \rightarrow 7 \rightarrow 8 \rightarrow 6 \rightarrow 11 \rightarrow 12 \rightarrow 9 \rightarrow 13 \rightarrow 10 \rightarrow 14 \rightarrow 4 \rightarrow 5 \rightarrow 3$} \\
\hline Correct Sign & Percentage of correct direction change predictions (PCDCP) & \multicolumn{2}{|c|}{$3 \rightarrow 5 \rightarrow 10 \& 12 \rightarrow 9 \rightarrow 14 \rightarrow 4 \rightarrow 13 \rightarrow 6 \rightarrow 8 \rightarrow 11 \rightarrow 1 \& 7 \rightarrow 2$} \\
\hline \multicolumn{4}{|c|}{$\begin{array}{l}{ }^{* 1} \mathrm{RW}\left[\text { Random Walk]; }{ }^{2} \mathrm{HM} \text { [Historical Mean]; }{ }^{3} \mathrm{SMA} 20 \text { [Simple Moving Average]; }{ }^{4} \mathrm{SMA} 60 ;{ }^{5} \mathrm{SES} \text { [Single Exponential Smoothing]; }{ }^{6} \mathrm{ARMA}(1,1)[\text { Auto Regressive Moving A }\right. \\
\text { [AutoRegressive]; }{ }^{8} \mathrm{AR}(5) ;{ }^{9} \mathrm{GARCH}(1,1) \text { [Generalized Auto Regressive Conditional Heteroscedasticity]; }{ }^{10} \mathrm{GARCH}-\mathrm{M}(1,1) ;{ }^{11} \mathrm{EGARCH}(1,1)\left[\text { Exponential GARCH]; }{ }^{12} \mathrm{TGARCH}(1\right. \\
\text { GARCH]; }{ }^{13} \mathrm{PARCH}(1,1) \text { [Power Auto Regressive Conditional Heteroscedasticity]; } ;{ }^{14} \mathrm{CGARCH}(1,1)[\text { Component GARCH] }\end{array}$} \\
\hline \multicolumn{4}{|c|}{ Table 2: Input- and Output-oriented Super-Efficiency Rankings } \\
\hline $\begin{array}{l}\text { Panel A: Combination } \\
\text { Output PCDCP in Inp }\end{array}$ & $\begin{array}{l}\text { of Performance Measures used as Inputs Along With } \\
\text { t-Oriented Analysis }\end{array}$ & $\begin{array}{l}\text { Panel B: Combination } \\
\text { Output PCDCP in Out }\end{array}$ & $\begin{array}{l}\text { Performance Measures used as Inputs Along With } \\
\text { t-Oriented Analysis }\end{array}$ \\
\hline Inputs & Models Ranked from Best to Worst & Inputs & Models Ranked from Best to Worst \\
\hline ME; MAE & $3 \rightarrow 5 \rightarrow 8 \rightarrow 6 \rightarrow 9 \rightarrow 10 \rightarrow 11 \rightarrow 12 \rightarrow 14 \rightarrow 7 \rightarrow 13 \rightarrow 4 \rightarrow 1 \rightarrow 2$ & ME; MAE & $3 \& 5 \& 8 \rightarrow 10 \& 12 \rightarrow 9 \rightarrow 14 \rightarrow 6 \rightarrow 4 \rightarrow 13 \rightarrow 11 \rightarrow 1 \rightarrow 7 \rightarrow 2$ \\
\hline ME; MAVolScE & $3 \rightarrow 5 \rightarrow 8 \rightarrow 6 \rightarrow 9 \& 10 \& 11 \& 12 \rightarrow 14 \rightarrow 7 \rightarrow 13 \rightarrow 4 \rightarrow 1 \rightarrow 2$ & ME; MAVolScE & $3 \& 5 \rightarrow 10 \& 12 \rightarrow 9 \rightarrow 14 \rightarrow 4 \rightarrow 8 \rightarrow 13 \rightarrow 6 \rightarrow 11 \rightarrow 1 \rightarrow 7 \rightarrow 2$ \\
\hline ME; MSE & $3 \rightarrow 14 \rightarrow 5 \rightarrow 11 \rightarrow 10 \rightarrow 12 \rightarrow 9 \rightarrow 13 \rightarrow 6 \rightarrow 4 \rightarrow 8 \rightarrow 7 \rightarrow 2 \rightarrow 1$ & ME; MSE & $3 \rightarrow 14 \rightarrow 5 \rightarrow 10 \rightarrow 12 \rightarrow 9 \rightarrow 13 \rightarrow 4 \rightarrow 6 \rightarrow 11 \rightarrow 8 \rightarrow 1 \& 7 \rightarrow 2$ \\
\hline ME; MSVolScE & $3 \rightarrow 14 \rightarrow 5 \rightarrow 11 \rightarrow 10 \rightarrow 12 \rightarrow 9 \rightarrow 13 \rightarrow 6 \rightarrow 4 \rightarrow 8 \rightarrow 7 \rightarrow 2 \rightarrow 1$ & ME; MSVolScE & $3 \rightarrow 14 \rightarrow 5 \rightarrow 10 \rightarrow 12 \rightarrow 9 \rightarrow 13 \rightarrow 4 \rightarrow 6 \rightarrow 11 \rightarrow 8 \rightarrow 1 \& 7 \rightarrow 2$ \\
\hline ME; MMEU & $3 \rightarrow 14 \rightarrow 5 \rightarrow 10 \rightarrow 13 \rightarrow 12 \rightarrow 9 \rightarrow 11 \rightarrow 4 \rightarrow 6 \rightarrow 8 \rightarrow 7 \rightarrow 2 \rightarrow 1$ & ME; MMEU & $3 \& 14 \rightarrow 5 \rightarrow 10 \& 12 \rightarrow 9 \rightarrow 4 \rightarrow 13 \rightarrow 6 \rightarrow 8 \rightarrow 11 \rightarrow 1 \rightarrow 7 \rightarrow 2$ \\
\hline ME; MMEO & $3 \rightarrow 1 \rightarrow 2 \rightarrow 11 \rightarrow 5 \rightarrow 8 \rightarrow 6 \rightarrow 9 \rightarrow 12 \rightarrow 10 \rightarrow 7 \rightarrow 14 \rightarrow 4 \rightarrow 13$ & ME; MMEO & $3 \rightarrow 2 \rightarrow 1 \rightarrow 11 \rightarrow 5 \rightarrow 8 \rightarrow 6 \rightarrow 9 \rightarrow 12 \rightarrow 10 \rightarrow 7 \rightarrow 14 \rightarrow 4 \rightarrow 13$ \\
\hline MVolScE; MAE & $3 \rightarrow 5 \rightarrow 8 \rightarrow 6 \rightarrow 9 \& 10 \rightarrow 11 \rightarrow 12 \rightarrow 14 \rightarrow 7 \rightarrow 13 \rightarrow 4 \rightarrow 1 \rightarrow 2$ & MVolScE; MAE & $3 \& 5 \& 8 \rightarrow 10 \& 12 \rightarrow 9 \rightarrow 14 \rightarrow 6 \rightarrow 4 \rightarrow 13 \rightarrow 11 \rightarrow 1 \& 7 \rightarrow 2$ \\
\hline MVolScE; MAVolScE & $3 \rightarrow 5 \rightarrow 8 \rightarrow 6 \rightarrow 9 \& 10 \& 11 \& 12 \rightarrow 14 \rightarrow 7 \rightarrow 13 \rightarrow 4 \rightarrow 1 \rightarrow 2$ & MVolScE; MAVolScE & $3 \& 5 \rightarrow 10 \& 12 \rightarrow 9 \rightarrow 14 \rightarrow 4 \rightarrow 8 \rightarrow 13 \rightarrow 6 \rightarrow 11 \rightarrow 1 \rightarrow 7 \rightarrow 2$ \\
\hline MVolScE; MSE & $3 \rightarrow 14 \rightarrow 5 \rightarrow 10 \rightarrow 12 \rightarrow 9 \rightarrow 11 \rightarrow 13 \rightarrow 6 \rightarrow 4 \rightarrow 8 \rightarrow 7 \rightarrow 2 \rightarrow 1$ & MVolScE; MSE & $3 \rightarrow 14 \rightarrow 5 \rightarrow 10 \rightarrow 12 \rightarrow 9 \rightarrow 13 \rightarrow 4 \rightarrow 6 \rightarrow 11 \rightarrow 8 \rightarrow 1 \& 7 \rightarrow 2$ \\
\hline MVolScE; MSVolScE & $3 \rightarrow 14 \rightarrow 5 \rightarrow 10 \rightarrow 12 \rightarrow 9 \rightarrow 11 \rightarrow 13 \rightarrow 6 \rightarrow 4 \rightarrow 8 \rightarrow 7 \rightarrow 2 \rightarrow 1$ & MVolScE; MSVolScE & $3 \rightarrow 14 \rightarrow 5 \rightarrow 10 \rightarrow 12 \rightarrow 9 \rightarrow 13 \rightarrow 4 \rightarrow 6 \rightarrow 11 \rightarrow 8 \rightarrow 1 \& 7 \rightarrow 2$ \\
\hline MVolScE; MMEU & $3 \rightarrow 14 \rightarrow 5 \rightarrow 10 \rightarrow 13 \rightarrow 12 \rightarrow 9 \rightarrow 11 \rightarrow 4 \rightarrow 6 \rightarrow 8 \rightarrow 7 \rightarrow 2 \rightarrow 1$ & MVolScE; MMEU & $3 \& 14 \rightarrow 5 \rightarrow 10 \& 12 \rightarrow 9 \rightarrow 4 \rightarrow 13 \rightarrow 6 \rightarrow 8 \rightarrow 11 \rightarrow 1 \rightarrow 7 \rightarrow 2$ \\
\hline MVolScE; MMEO & $3 \rightarrow 1 \rightarrow 2 \rightarrow 11 \rightarrow 5 \rightarrow 8 \rightarrow 6 \rightarrow 12 \rightarrow 9 \rightarrow 10 \rightarrow 14 \rightarrow 7 \rightarrow 4 \rightarrow 13$ & MVolScE; MMEO & $3 \rightarrow 2 \rightarrow 1 \rightarrow 11 \rightarrow 5 \rightarrow 8 \rightarrow 6 \rightarrow 12 \rightarrow 9 \rightarrow 10 \rightarrow 7 \rightarrow 14 \rightarrow 4 \rightarrow 13$ \\
\hline
\end{tabular}

${ }^{* 1} \mathrm{RW} ;{ }^{2} \mathrm{HM} ;{ }^{3} \mathrm{SMA} 20 ;{ }^{4} \mathrm{SMA} 60 ;{ }^{5} \mathrm{SES} ;{ }^{6} \operatorname{ARMA}(1,1) ;{ }^{7} \operatorname{AR}(1) ;{ }^{8} \mathrm{AR}(5) ;{ }^{9} \operatorname{GARCH}(1,1) ;{ }^{10} \operatorname{GARCH}-\mathrm{M}(1,1) ;{ }^{11} \operatorname{EGARCH}(1,1) ;{ }^{12} \mathrm{TGARCH}(1,1) ;{ }^{13} \mathrm{PARCH}(1,1) ;{ }^{14} \mathrm{CGARCH}(1,1)$ 
Table 3: Efficient Frontiers With Different Performance Levels

\begin{tabular}{|c|c|c|c|c|c|c|c|c|c|c|}
\hline \multirow[b]{2}{*}{$\begin{array}{c}\text { Efficient } \\
\text { Frontiers }\end{array}$} & \multicolumn{5}{|c|}{$\begin{array}{l}\text { Panel A: Combinations of Performance Measures used as Inputs Along } \\
\text { With Output PCDCP }\end{array}$} & \multicolumn{5}{|c|}{$\begin{array}{l}\text { Panel B: Combinations of Performance Measures used as Inputs Along With } \\
\text { Output PCDCP }\end{array}$} \\
\hline & $\begin{array}{l}\text { ME \& } \\
\text { MAE }\end{array}$ & $\begin{array}{l}\text { ME \& } \\
\text { MAVolScE }\end{array}$ & $\begin{array}{c}\text { ME \& MSE; } \\
\text { ME \& } \\
\text { MSVolScE }\end{array}$ & $\begin{array}{l}\text { ME \& } \\
\text { MMEU }\end{array}$ & ME \& MMEO & $\begin{array}{l}\text { MVolScE } \\
\text { \& MAE }\end{array}$ & $\begin{array}{l}\text { MVolScE \& } \\
\text { MAVolScE }\end{array}$ & $\begin{array}{c}\text { MVolScE \& } \\
\text { MSE; MVolScE } \\
\text { \& MSVolScE }\end{array}$ & $\begin{array}{l}\text { MVolScE } \\
\text { \& MMEU }\end{array}$ & $\begin{array}{l}\text { MVolScE \& } \\
\text { MMEO }\end{array}$ \\
\hline$E^{l}$ & $\{3,5,8\}$ & $\{3,5\}$ & $\{3,5,14\}$ & $\{3,14\}$ & $\{1,2,3,5,6,8,11\}$ & $\{3,5,8\}$ & $\{3,5\}$ & $\{3,5,14\}$ & $\{3,14\}$ & $\{1,2,3,5,6,8,11\}$ \\
\hline$E^{2}$ & $\{6,9,10,11\}$ & $\{6,8,9,10,11\}$ & $\{9,10,11,13\}$ & $\{5\}$ & $\{7,9,1012\}$ & $\{6,11\}$ & $\{6,8,10,12\}$ & $\{10,13\}$ & $\{5\}$ & $\{7,12\}$ \\
\hline$E^{3}$ & $\{12,14\}$ & $\{12,14\}$ & $\{12\}$ & $\{9,10,11\}$ & $\{13,14\}$ & $\{9,12\}$ & $\{9\}$ & $\{12\}$ & $\{10\}$ & $\{9,10\}$ \\
\hline$E^{4}$ & $\{4,7,13\}$ & $\{4,7,13\}$ & $\{4,6\}$ & $\{12,13\}$ & $\{4\}$ & $\{4,7,13\}$ & $\{11,14\}$ & $\{9,11\}$ & $\{12,13\}$ & $\{13,14\}$ \\
\hline$E^{5}$ & $\{1,2\}$ & $\{1,2\}$ & $\{2,8\}$ & $\{4\}$ & & $\{1,2\}$ & $\{4,7,13\}$ & $\{4,6\}$ & $\{9\}$ & $\{4\}$ \\
\hline$E^{6}$ & & & $\{7\}$ & $\{6\}$ & & & $\{1,2\}$ & $\{2,8\}$ & $\{4,11\}$ & \\
\hline$E^{7}$ & & & $\{1\}$ & $\{2,8\}$ & & & & $\{7\}$ & $\{6\}$ & \\
\hline$E^{8}$ & & & & $\{7\}$ & & & & $\{1\}$ & $\{2,8\}$ & \\
\hline$E^{9}$ & & & & $\{1\}$ & & & & & $\{7\}$ & \\
\hline$E^{10}$ & & & & & & & & & $\{1\}$ & \\
\hline
\end{tabular}

Table 4: SBM-CDEA Rankings

\begin{tabular}{ll|ll}
\hline $\begin{array}{l}\text { Panel A: Combinations of Performance Measures used as Inputs Along With } \\
\text { Output PCDCP }\end{array}$ & \multicolumn{1}{l}{$\begin{array}{l}\text { Panel B: Combinations of Performance Measures used as Inputs Along With Output } \\
\text { PCDCP }\end{array}$} \\
\hline \multicolumn{1}{c|}{ Inputs } & \multicolumn{1}{c}{ Models Ranked from Best to Worst } & \multicolumn{1}{c}{ Inputs } \\
\hline ME; MAE & $3 \rightarrow 5 \rightarrow 8 \rightarrow 6 \rightarrow 9 \& 10 \rightarrow 11 \rightarrow 12 \rightarrow 14 \rightarrow 13 \rightarrow 4 \rightarrow 7 \rightarrow 2 \rightarrow 1$ & MVolScE; MAE & $3 \rightarrow 5 \rightarrow 8 \rightarrow 6 \rightarrow 10 \rightarrow 9 \rightarrow 12 \rightarrow 14 \rightarrow 11 \rightarrow 13 \rightarrow 4 \rightarrow 7 \rightarrow 2 \rightarrow 1$ \\
ME; MAVolScE & $3 \rightarrow 5 \rightarrow 11 \rightarrow 9 \rightarrow 10 \rightarrow 6 \rightarrow 8 \rightarrow 14 \rightarrow 12 \rightarrow 4 \rightarrow 13 \rightarrow 7 \rightarrow 2 \rightarrow 1$ & MVolScE; MAVolScE & $3 \rightarrow 5 \rightarrow 10 \& 12 \rightarrow 6 \rightarrow 8 \rightarrow 9 \rightarrow 14 \rightarrow 11 \rightarrow 4 \rightarrow 13 \rightarrow 7 \rightarrow 2 \rightarrow 1$ \\
ME; MSE & $3 \rightarrow 5 \rightarrow 14 \rightarrow 9 \rightarrow 11 \rightarrow 10 \rightarrow 13 \rightarrow 12 \rightarrow 4 \rightarrow 6 \rightarrow 8 \rightarrow 2 \rightarrow 7 \rightarrow 1$ & MVolScE; MSE & $3 \rightarrow 5 \rightarrow 14 \rightarrow 10 \rightarrow 13 \rightarrow 12 \rightarrow 9 \rightarrow 11 \rightarrow 4 \rightarrow 6 \rightarrow 8 \rightarrow 2 \rightarrow 7 \rightarrow 1$ \\
ME; MSVolScE & $3 \rightarrow 5 \rightarrow 14 \rightarrow 9 \rightarrow 11 \rightarrow 10 \rightarrow 13 \rightarrow 12 \rightarrow 4 \rightarrow 6 \rightarrow 8 \rightarrow 2 \rightarrow 7 \rightarrow 1$ & MVolScE; MSVolScE & $3 \rightarrow 5 \rightarrow 14 \rightarrow 10 \rightarrow 13 \rightarrow 12 \rightarrow 9 \rightarrow 11 \rightarrow 4 \rightarrow 6 \rightarrow 8 \rightarrow 2 \rightarrow 7 \rightarrow 1$ \\
ME; MMEU & $3 \rightarrow 14 \rightarrow 5 \rightarrow 9 \rightarrow 10 \rightarrow 11 \rightarrow 12 \rightarrow 13 \rightarrow 4 \rightarrow 6 \rightarrow 8 \rightarrow 2 \rightarrow 7 \rightarrow 1$ & MVolScE; MMEU & $3 \rightarrow 14 \rightarrow 5 \rightarrow 10 \rightarrow 12 \rightarrow 13 \rightarrow 9 \rightarrow 11 \rightarrow 4 \rightarrow 6 \rightarrow 8 \rightarrow 2 \rightarrow 7 \rightarrow 1$ \\
ME; MMEO & $3 \rightarrow 5 \rightarrow 2 \rightarrow 11 \rightarrow 1 \rightarrow 6 \rightarrow 8 \rightarrow 9,10 \& 12 \rightarrow 14 \rightarrow 13 \rightarrow 4$ & MVolScE; MMEO & $3 \rightarrow 5 \rightarrow 2 \rightarrow 1 \rightarrow 11 \rightarrow 6 \rightarrow 8 \rightarrow 12 \rightarrow 7 \rightarrow 9 \& 10 \rightarrow 14 \rightarrow 13 \rightarrow 4$ \\
\hline${ }^{* 1} \mathrm{RW} \cdot{ }^{2} \mathrm{HM} \cdot{ }^{3} \mathrm{SMA} 20 \cdot{ }^{4} \mathrm{SMA} 60 \cdot{ }^{5} \mathrm{SES} ;{ }^{6} \mathrm{ARMA}(1,1) \cdot{ }^{7} \mathrm{AR}(1) \cdot{ }^{8} \mathrm{AR}(5) \cdot{ }^{9} \mathrm{GARCH}(1,1) \cdot{ }^{10} \mathrm{GARCH}-\mathrm{M}(1,1) \cdot{ }^{11} \mathrm{EGARCH}(1,1) \cdot{ }^{12} \mathrm{TGARCH}(1,1) \cdot{ }^{13} \mathrm{PARCH}(1,1) \cdot{ }^{14} \mathrm{CGARCH}(1,1)$
\end{tabular}

$*{ }^{*} \mathrm{RW} ;{ }^{2} \mathrm{HM} ;{ }^{3} \mathrm{SMA} 20 ;{ }^{4} \mathrm{SMA} 60 ;{ }^{5} \mathrm{SES} ;{ }^{6} \mathrm{ARMA}(1,1) ;{ }^{7} \mathrm{AR}(1) ;{ }^{8} \mathrm{AR}(5) ;{ }^{9} \operatorname{GARCH}(1,1) ;{ }^{10} \operatorname{GARCH}-\mathrm{M}(1,1) ;{ }^{11} \operatorname{EgARCH}(1,1) ;{ }^{12} \operatorname{TGARCH}(1,1) ;{ }^{13} \mathrm{PARCH}(1,1) ;{ }^{14} \mathrm{CGARCH}(1,1)$ 


\section{CONCLUSION}

$\mathrm{Xu}$ and Ouenniche (2012a) proposed an input-oriented radial super-efficiency DEA-based framework to evaluate the performance of competing forecasting models of crude oil prices' volatility, which delivers a single ranking based on multiple performance criteria; such a framework suffers from several issues that were overcome in this paper. The main results may be summarized as follows. First, models that are on the efficient frontier and have zero slacks regardless of the performance measures used (e.g., SMA20) maintain their ranks regardless of the choice of DEA analysis and its orientation. Second, the multicriteria rankings of the best and the worst models seem to be relatively robust to changes in most performance measures; in sum, SMA20 is the best across the board and, for the remaining models, differences in rankings were mainly due to the presence of slacks and the nature of benchmarks. Finally, when under-estimated forecasts are penalized, most GARCH types of models tend to perform well suggesting that they often produce forecasts that are over-estimated. On the other hand, when over-estimated forecasts are penalized, averaging models such as RW, HM, SES tend to perform very well - suggesting that these models often produce forecasts that are under-estimated.

\section{AUTHOR INFORMATION}

Dr. Jamal Ouenniche is a Reader in Management Science at the University of Edinburgh, Business School, Edinburgh, UK. His research focuses on the design and implementation of mathematical programming-based and artificial intelligence-based methods. His papers have been published in journals such as Operation Research, European Journal of Operational Research, and International Journal of Production Research amongst others. E-mail: Jamal.Ouenniche@ed.ac.uk (Corresponding author)

Dr. Bing Xu is a Lecturer at the School of Management \& Languages; Accountancy, Economics and Finance; Heriot-Watt University; Edinburgh; EH14 4AS, UK. Her papers have been published in Expert Systems with Applications, Energy Economics, and Applied Financial Economics amongst others. E-mail: b.xu@hw.ac.uk

Professor Kaoru Tone is Professor Emeritus at the National Graduate Institute for Policy Studies, Tokyo, Japan. His papers have been published in European Journal of Operational Research, Journal of the Operational Research Society, Omega, International Transactions in Operational Research, and Annals of Operations Research amongst others.

\section{REFERENCES}

1. Banker, R. D., Cooper, W. W., Seiford, L. M., Thrall, R. M., \& Zhu, J. (2004). Returns to scale in different DEA models. European Journal of Operational Research, 154, 345-362.

2. Majid, S.-D. (2012). On a basic definition of returns to scale. Operations Research Letters, 40, 144-147.

3. Morita, H., Hirokawa, K., \& Zhu, J. (2005). A slack-based measure of efficiency in context-dependent data envelopment analysis. Omega, 33, 357-362.

4. Seiford, L. M., \& Zhu, J. (2003). Context-dependent data envelopment analysis - measuring attractiveness and progress. Omega, 31, 397-408.

5. Tone, K. (2001). A slacks-based measure of efficiency in data envelopment analysis. European Journal of Operational Research, 130, 498-509.

6. Xu, B., \& Ouenniche, J. (2011). A multidimensional framework for performance evaluation of forecasting models: context-dependent DEA. Applied Financial Economics, 21, 1873-1890.

7. Xu, B., \& Ouenniche, J. (2012a). A data envelopment analysis-based framework for the relative performance evaluation of competing crude oil prices' volatility forecasting models. Energy Economics, 34, 576-583.

8. Xu, B., \& Ouenniche, J. (2012b). Performance evaluation of competing forecasting models: A multidimensional framework based on MCDA. Expert Systems with Applications, 39, 8312-8324. 


\section{$\underline{\text { NOTES }}$}

